2. Page HL, Engel HJ, Campbell WB, et al. Anomalous origin of the left circumflex coronary artery: recognition, angiographic demonstration and clinical significance. Circulation. 1974;50:768-73.

3. Wilkins CE, Betancourt B, Mathur VS, et al. Coronary artery anomalies: a review of more than 10,000 patients from the Clayton Cardiovascular Laboratories. Tex Heart Inst J. 1988;15:166-73.

4. Morino Y, Abe M, Morimoto T, et al. J CTO Registry Investigators. Predicting successful guidewire crossing through chronic total occlusion of native coronary lesions within 30 minutes: the J-CTO (Multicenter CTO Registry in Japan) score as a difficulty grading and time assessment tool. JACC Cardiovasc Interv. 2011;4:213-21.

5. Mohsen GA, Mohsin KG, Forsberg M, et al. Anomalous left circumflex artery from the right coronary cusp: a benign variant? Invasive Cardiol. 2013;25:284-7.

6. Morgan KP, Morris GM, Al-Najjar Y, et al. Percutaneous intervention on anomalous circumflex coronary arteries: a single centre experience. Cardiovasc Revasc Med. 2012;13: 335-40.

7. Suero JA, Marso SP, Jones PG, et al. Procedural outcomes and long-term survival among patients undergoing percutaneous coronary intervention of a chronic total occlusion in native coronary arteries: a 20-year experience. J Am Coll Cardiol. 2001;38:409-14.

8. Jolly SS, Amlani S, Hamon M, et al. Radial versus femoral access for coronary angiography or intervention and the impact on major bleeding and ischemic events: a systematic review and metaanalysis of randomized trials. Am Heart J. 2009;157:132-40.

Javier Benezet*, Antonio Agarrado,Jesús Oneto

Department of Cardiology, Hospital de Jerez de la Frontera, Jerez de la Frontera, Cádiz, Spain

* Corresponding author at: Servicio de Cardiología, Hospital de Jerez de la Frontera, Circunvalación s/n, 11407 Jerez de la Frontera, Cádiz, Spain.

E-mail address: javbenezet@hotmail.com (J. Benezet).

http://dx.doi.org/10.1016/j.acmx.2016.06.007 1405-9940/

(c) 2016 Instituto Nacional de Cardiología Ignacio Chávez. Published by Masson Doyma México S.A. This is an open access article under the CC BY-NC-ND license (http://creativecommons.org/licenses/ by-nc-nd/4.0/).

\section{Manejo de la mediastinitis postrasplante cardiaco mediante mediastinoscopia y perlas antibióticas de liberación prolongada}

\section{Dissolvable and extended release antibiotic beads in mediastinoscopic management of mediastinitis after heart transplantation}

Las complicaciones quirúrgicas relacionadas con la esternotomía media se pueden presentar en los pacientes postoperados de cirugía cardiaca como un amplio espectro de afecciones, que varían desde la dehiscencia de la herida quirúrgica hasta la mediastinitis supurativa. De este modo, los términos esternitis, mediastinitis, complicación de la herida e infección de la herida quirúrgica han sido utilizados indistintamente para denotar infección mediastinal profunda ${ }^{1}$.

En aquellos pacientes sometidos a trasplante cardiaco (TC), la mediastinitis posquirúrgica constituye una complicación grave y de difícil diagnóstico, que pone en riesgo la vida de los pacientes; además, muestra aspectos únicos debido al estado de inmunosupresión en el que estos se encuentran ${ }^{2}$.

Una vez establecido el diagnóstico, debe instituirse el tratamiento de inmediato, generalmente combinando antibioticoterapia con cirugía, lavado y desbridamiento intensivos. Por desgracia, el tratamiento convencional tiene varias desventajas, tales como la inestabilidad de la caja torácica, la inmovilización prolongada y el trauma quirúrgico sustancialmente mayor, que puede ser perjudicial ${ }^{3,4}$.

Al respecto, se ha descrito un gran número de abordajes quirúrgicos para el manejo de la mediastinitis; sin embargo, el tratamiento quirúrgico óptimo continúa siendo controversial. Basándose en los hallazgos tomográficos y los datos clínicos, resulta posible realizar incisiones y drenajes por vía transcervical, subxifoidea y toracoscópica y por toracotomía y mediastinoscopia videoasistida ${ }^{3}$.

El otro punto angular del tratamiento es la terapia con antibióticos. Uno de sus principales objetivos consiste en alcanzar altas concentraciones farmacológicas en el sitio de infección. Stimulan ${ }^{\circledR}$ (Biocomposites Ltd., Keele, Staffordshire, Reino Unido) es un medio absorbible para la liberación de antibióticos a nivel local y que ha probado su eficacia con anterioridad en la cirugía ortopédica, tanto en la profilaxis como en el tratamiento de la osteomielitis ${ }^{5}$.

A continuación presentamos nuestra experiencia con 2 casos de mediastinitis manejados mediante procedimiento de mínima invasión y colocación de perlas de liberación de antibióticos en pacientes sometidos a TC.

Se trató de 2 pacientes masculinos, ambos con antecedente de TC y bajo tratamiento inmunosupresor (a base de ácido micofenólico, prednisona y sirolimus), de 42 y 62 años de edad y con diagnóstico establecido de mediastinitis tipo IIIb y v de Oakley ${ }^{1}$, respectivamente.

Con base en los hallazgos tomográficos de ambos casos (fig. 1a b) se optó por realizar abordaje subxifoideo y mediastinoscopia (fig. 1c). Se realizó drenaje de las colecciones, lavado con abundante solución salina y desbridación del tejido infectado; además, se tomaron muestras de tejido y del exudado para cultivo. Se utilizó después del lavado instrumental limpio para la liberación in situ de antibióticos, con cambio de batas, campos y guantes para todo el equipo quirúrgico.

Posteriormente, se colocaron perlas de sulfato de calcio con antibiótico para liberación in situ (Stimulan ${ }^{\circledR}$ ); en el primer caso se utilizó imipenem/amikacina, y en el segundo paciente, imipenem/vancomicina y clindamicina (fig. 1d). Al final de la cirugía se dejó un sistema de drenaje cerrado a 


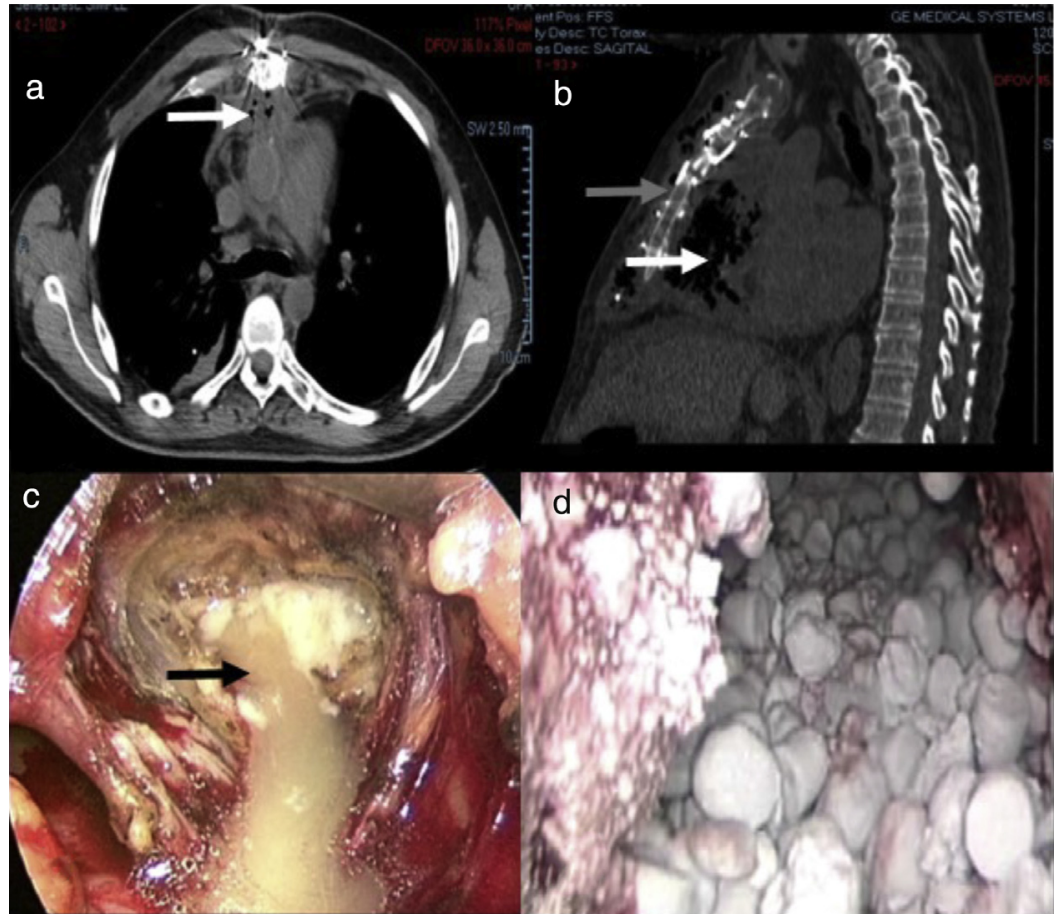

Figura 1 Tomografía axial computarizada; cortes axial (1 a) y sagital (1 b); se observa la presencia de gas (flecha blanca) mediastinal y enfisema subcutáneo (flecha gris). Vista mediastinoscópica $(1 \mathrm{c})$ : nótese la salida de material purulento una vez realizado el abordaje quirúrgico (flecha negra). Vista mediastinoscópica (1 d) en donde se aprecian las perlas de sulfato de calcio con antibióticos para liberación prolongada in situ.

derivación (Drenovac, S. A. de c. v.), que se retiró al reducir los gastos a menos de $40 \mathrm{cc} / 24 \mathrm{~h}$.

Las perlas fueron preparadas transoperatoriamente mediante la mezcla del antibiótico seleccionado junto con el polvo de Stimulan ${ }^{\circledR}$. Las dosis recomendadas dependen del tipo de antibiótico ${ }^{5-11}$ (tabla 1). Dicha solución fue posteriormente mezclada durante aproximadamente $30 \mathrm{~s}$, y la mezcla se aplicó en una estera para perlas, en donde se colocó en un periodo de tiempo de 3-5 min. Después de la colocación, las perlas fueron retiradas de la estera y se aplicaron en el sitio seleccionado ${ }^{5}$.

Ambos pacientes fueron extubados en el quirófano, inmediatamente después de la cirugía, y ninguno de los 2 requirió apoyo con aminas durante la cirugía ni durante su estancia en Terapia Postquirúrgica. Los drenajes fueron retirados a las $48 \mathrm{~h}$ postoperatorias y egresaron a piso a las $72 \mathrm{~h}$ de la cirugía, por buena evolución clínica, finalizando su esquema antimicrobiano y siendo dados de alta por mejoría. Tanto los valores de laboratorio como el recuento de leucocitos, linfocitos, reactantes de fase aguda y procalcitonina al egreso fueron normales, mientras que las tomografías de control únicamente mostraron cambios posquirúrgicos.

En el seguimiento a 3 meses no se presentó ninguna recidiva y los 2 pacientes se han reintegrado a su vida y actividades diarias. La incidencia de complicaciones relacionadas con la herida quirúrgica en los receptores de TC se presenta en un 8 al $15 \%$ de los casos, aunque se han reportado incidencias tan altas como de un $40 \%$. A este respecto, las heridas superficiales varían entre 3.9 y $16 \%$ de los receptores de TC, mientras que las infecciones profundas, incluyendo mediastinitis, tienen una incidencia de 2.4 a $35 \%$. Por otra parte, la dehiscencia esternal ocurre en un $12.5 \%$ a $25 \%$ de los receptores de $\mathrm{TC}^{6}$.

Con el fin de unificar criterios y determinar el mejor tratamiento se han utilizado las siguientes definiciones: 1) dehiscencia mediastinal: apertura de la esternotomía en ausencia de evidencia de infección clínica o microbiológica; 2) infección de herida mediastinal: evidencia clínica o microbiológica de tejido preesternal y osteomielitis esternal, con o sin sepsis mediastinal y con o sin inestabilidad esternal; los subtipos incluyen; A) infección superficial de la herida, confinada a tejido celular subcutáneo; B) infección profunda de la herida (mediastinitis), y finalmente, $C$ ) infección de la herida asociada con osteomielitis esternal con o sin espacio restroesternal infectado ${ }^{1}$.

Al hablar de TC y mediastinitis postesternotomía se ha observado que la incidencia es al menos 2 veces superior después de TC que en pacientes sometidos a cirugía cardiaca convencional ${ }^{2}$.

Los gérmenes más comúnmente aislados en estos casos también son grampositivos (especialmente Staphylococcus aureus), generalmente resistentes a meticilina, así como Enterococcus faecalis; además, se han aislado gramnegativos, como Escherichia coli y Acinetobacter. Notablemente, se ha descrito una incidencia mayor de infecciones por hongos en receptores de TC con respecto de otras cirugías cardiacas $^{6}$.

Sin embargo, la mayoría de los estudios de mediastinitis han examinado a pacientes que no han recibido terapia de inducción (aproximadamente 2.5-7.5\% de los pacientes postoperados de TC). Y varios investigadores han postulado la teoría de que es más probable un incremento en la tasa 
de infecciones en aquellos pacientes inmunocomprometidos a los cuales se les realizará $\mathrm{TC}^{2}$.

Los factores de riesgo independientes para desarrollar complicaciones relacionadas con la herida quirúrgica en TC resultan más controversiales que para cirugía cardiaca convencional. Se acepta que algunos comúnmente mencionados, como son la diabetes mellitus, las reoperaciones, la ventilación mecánica asistida, la estancia hospitalaria prolongada y el incremento en el IMC, son de relevancia en estos pacientes. Sin embargo, se consideran también aquellos factores propios de TC como más relevantes, como el uso de un esquema específico de inmunosupresión y un deterioro en el estado general de salud previo a la recepción del injerto, la falla renal y el antecedente de DAV, como puente a trasplante ${ }^{2,6}$.

Para establecer el diagnóstico de mediastinitis en los receptores de $\mathrm{TC}$, especialmente en aquellos en los cuales existe algún factor de riesgo adicional para complicaciones relacionadas con la herida quirúrgica, se necesita un alto nivel de precaución. El abordaje deberá llevarse a cabo por un equipo multidisciplinario y utilizar las herramientas diagnósticas adecuadas, que permitan establecer un diagnóstico temprano de mediastinitis ${ }^{6}$.

Clínicamente, los síntomas de mediastinitis pueden diferir en los receptores de TC al compararse con otras cirugías. La inmunosupresión (incluyendo el uso de esteroides) puede cambiar los signos típicos de infección, y los signos locales, como eritema y exudado purulento, pueden estar ausentes. La fiebre (definida como una temperatura $>38^{\circ} \mathrm{C}$ ) está presente en el $30 \%$ de los casos, mientras que la leucocitosis se presenta en el $40 \%$ de los pacientes con mediastinitis y TC, siendo el síntoma más común el dolor torácico retroesternal (60\%) con desproporción respecto a los hallazgos clínicos; por otro lado, el exudado y el eritema solo están presentes en el $33 \%$ de los casos $^{6}$.

La tomografía axial computarizada muestra como hallazgos aire y/o colecciones hasta en el $60 \%$ de los pacientes con infección profunda, mientras que la radiografía de tórax solo apoyará el diagnóstico con base en los hallazgos clínicos. La aspiración con aguja guiada por tomografía axial computarizada puede ser útil para confirmar el diagnóstico ${ }^{6}$.

Existe un consenso de que, una vez establecido el diagnóstico de mediastinitis, el tratamiento quirúrgico intensivo es esencial, y que debe realizarse desbridamiento, incluyendo la escisión radical del tejido necrótico. Aparte de esta acción inicial, las opciones recomendadas para el manejo quirúrgico óptimo difieren. Se ha reportado el desbridamiento intensivo y a continuación irrigación subesternal con Isodine ${ }^{\circledR}$, con cierre primario ${ }^{8}$, así como una serie de 15 pacientes con mediastinitis postrasplante, manejada exitosamente mediante drenaje cerrado ${ }^{9}$. Y un protocolo de manejo con tórax abierto, que involucra desbridamiento intensivo del tejido necrótico y drenaje con VAC, acompañado por, al menos, 6 semanas con antibióticos IV ${ }^{10}$.

En el año 2006, Shimizu et al. realizaron el primer drenaje por mediastinoscopia videoasistida, en una paciente con diagnóstico de mediastinitis, demostrando que esta técnica es segura, que permitía la manipulación bimanual, con inserción de varios instrumentos y una excelente visualización, lo cual facilitaba el drenaje y el desbridamiento de los abscesos mediastinales. Es un procedimiento sencillo en pacientes seleccionados y no requiere de intubación selectiva, como en el caso de la toracoscopia o la toracotomía, lo cual evita el riesgo de empiema ${ }^{3}$.

El otro hecho a considerar es que la administración sistémica de antibióticos puede causar toxicidad a altas dosis; y he aquí por qué la liberación local de antibióticos resulta una opción útil. El sistema de liberación local ideal debe ser capaz de proporcionar una dosis adecuada para combatir la infección y también ser biodegradable, con el fin de evitar otro procedimiento quirúrgico al retirarse ${ }^{5}$.

El sulfato de calcio - $\mathrm{CaSO}_{4}$ - es un químico común industrial y de laboratorio; ha sido utilizado en cirugía ortopédica durante varios años. Al mezclar el polvo de hemihidrato con agua, se consigue la formación de dihidrato de calcio $-\mathrm{CaSO}_{4} 2 \mathrm{H}_{2} \mathrm{O}-$, el cual puede ser moldeado en perlas. Combinar los antibióticos con dicho polvo resulta en la formación de perlas cargadas con antibióticos. El antibiótico es liberado lentamente a medida que las perlas son reabsorbidas $^{5,11}$. Una gran cantidad de antibióticos puede combinarse con el sulfato de calcio, incluyendo vancomicina, tobramicina, teicoplanina, cefazolina y fucidina (ver tabla 1). El tiempo de preparación depende del lapso que tarda en convertirse el hemihidrato en dihidrato; y dicho tiempo varía con los antibióticos empleados. Además, pueden combinarse 2 antibióticos simultáneamente $e^{5,11}$.

Stimulan $^{\circledR}$ es un producto sintético a base de sulfato de calcio, biodegradable y que se absorbe completamente in vivo. Puesto que es preparado sintéticamente, no contiene las impurezas que podrían presentar las formas naturales de sulfato de calcio. Se absorbe completamente en 3 o 4 semanas, y permite la liberación completa de los antibióticos ${ }^{5}$.

El volumen de las perlas utilizadas varía entre 5 y $70 \mathrm{cc}$, con un promedio de $20 \mathrm{cc}$, y se ha documentado previamente el uso de Stimulan ${ }^{\circledR}$ en el tratamiento de la osteomielitis crónica en extremidades inferiores, con unas tasas de resolución de la infección de hasta el 93\% de los casos $^{5}$.

Como se ha comentado anteriormente, el diagnóstico temprano y la intervención oportuna son determinantes para mejorar la sobrevida y el pronóstico. El manejo tradicional incluye desbridamiento de la herida, irrigación con antibióticos y valoración del uso de epiplón y/o superposición de músculos pectorales ${ }^{2,6}$. El tratamiento con mediastinoscopia, por otra parte, cumple con estas recomendaciones, reduce la extensión del área cruenta, sustituye la irrigación por la aplicación de cápsulas para la liberación local de antibiótico, y además ocasiona una menor respuesta inflamatoria y acorta la recuperación posquirúrgica.

Como conclusiones, podemos decir que la mediastinitis postesternotomía es una complicación grave, con una morbimortalidad alta y de difícil manejo, cuyas consecuencias generalmente son devastadoras cuando se presentan.

Los receptores de TC representan un grupo especial de pacientes inmunosuprimidos en los cuales la mediastinitis muestra aspectos únicos y cuyo tratamiento tradicional implica un manejo quirúrgico bastante intensivo, el cual la mayoría de las veces conlleva un alto índice de recidivas y complicaciones. 
Tabla 1 Combinaciones y dosis recomendadas de antibióticos

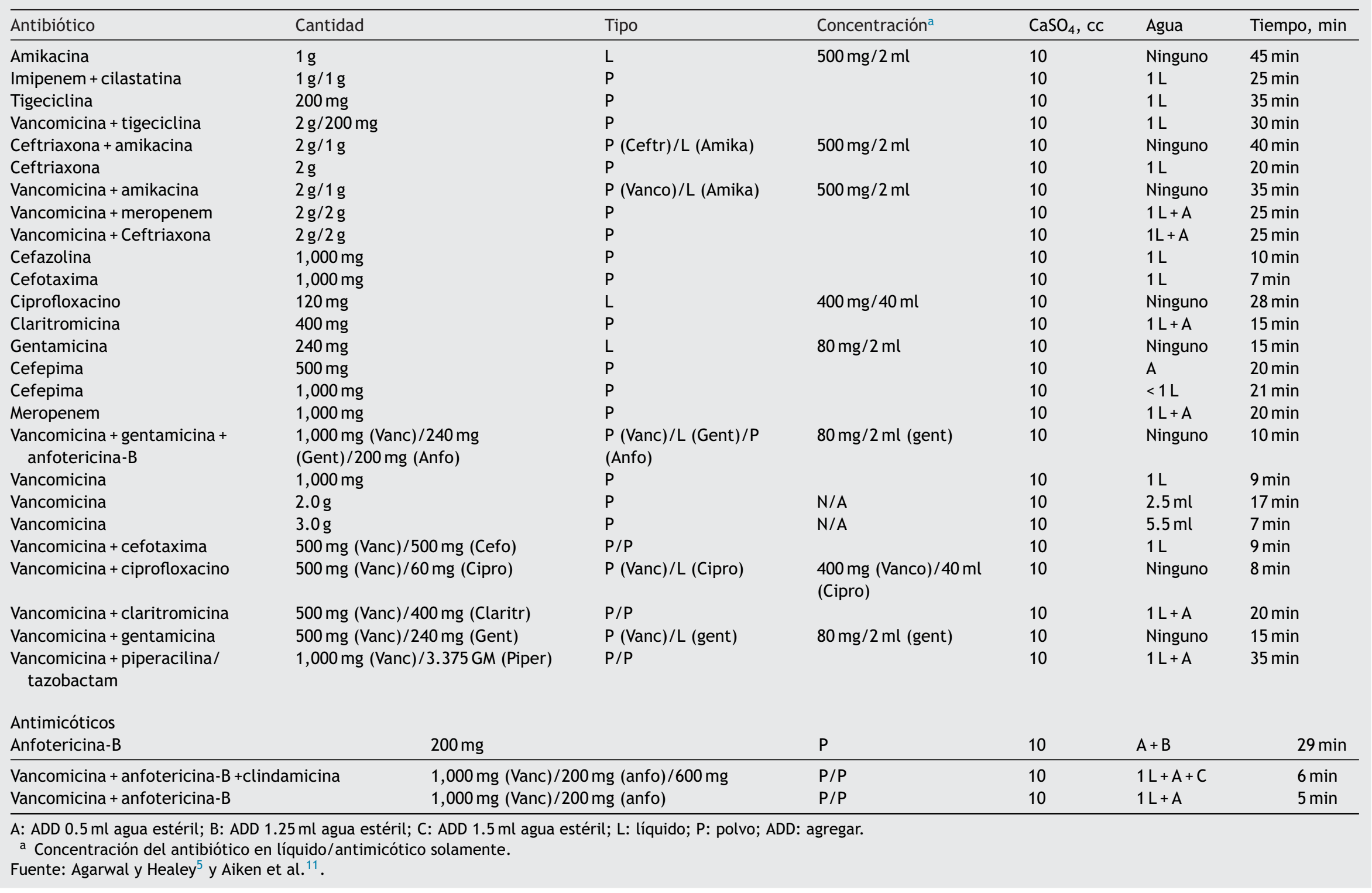


Consideramos que esta modalidad terapéutica puede ser una alternativa en el manejo de la mediastinitis postesternotomía en pacientes seleccionados y que es necesario realizar más estudios al respecto, involucrando un mayor número de pacientes, con la finalidad de determinar la efectividad de esta modalidad terapéutica.

\section{Bibliografía}

1. El Oakley RM, Wright JE. Postoperative mediastinitis: Classification and management. Ann Thorac Surg. 1996;61:1030-6.

2. Chou NK, Wang JL, Chi NH, et al. Surgical treatment of mediastinitis after cardiac transplantation. Trasplant Proc. 2008;40:2629-30.

3. Shimizu K, Otani Y, Nakano T, et al. Successful video-assisted mediastinoscopic drainage of descending necrotizing mediastinitis. Ann Thorac Surg. 2006;81:2279-81.

4. Sjögren J, Gustafsson R, Nilsson J, et al. Clinical outcome after poststernotomy mediastinitis: Vacuum-assisted closure versus conventional treatment. Ann Thorac Surg. 2005;79: 2049-55.

5. Agarwal S, Healey B. The use of antibiotic impregnated absorbable calcium sulphate beads in management of infected joint replacement prostheses. JAJS. 2014;1:72-5.

6. Zuckermann A, Barten M. Surgical wound complications after heart transplantation. Transpl Int. 2011;24:627-36.

7. Fernández-de la Reguera G, Soto-Nieto G, López-Madrigal N, et al. Mediastinitis posquirúrgica. Arch Cardiol Mex. 2011;81 Supl 2:64-72.

8. Abid Q, Nkere UU, Hasan A, et al. Mediastinitis in heart and lung transplantation: 15 years experience. Ann Thorac Surg. 2003;75:1565-71.

9. Senechal M, LePrince P, Tezenas du Montcel S, et al. Bacterial mediastinitis after heart transplantation: Clinical presentation, risk factors and treatment. J Heart Lung Transplant. 2004;23:165-70.
10. Filosoufi F, Rahmanian PB, Castillo JG, et al. Incidence, treatment strategies and outcome of deep sternal wound infection after orthotopic heart transplantation. J Heart Lung Transplant. 2007;26:1084-90.

11. Aiken SS, Cooper JJ, Florance H, et al. Local release of antibiotics for surgical site infection management using high-purity calcium sulfate: An in vitro elution study. Surg Infec (Larchmt). 2015;16:54-61.

Luis Raúl Meza-López ${ }^{\mathrm{a}, *}$, José Angel Cigarroa-López ${ }^{\mathrm{b}}$, Silvia Hernández-Meneses ${ }^{a}$, David Arturo Castán-Flores ${ }^{b}$, Genaro Hiram Mendoza-Zavala ${ }^{\mathrm{b}}$, José Antonio Barragán-Zamora ${ }^{a}$, Arturo Carrillo-Muñoz y Daniel Alejandro Munguía-Canales ${ }^{a}$

a Departamento de Cirugía Cardiotorácica, UMAE Hospital de Cardiología del Centro Médico Nacional Siglo XXI, Instituto Mexicano del Seguro Social, Ciudad de México, México

${ }^{\mathrm{b}}$ Clínica de Insuficiencia Cardiaca y Trasplante, UMAE Hospital de Cardiología del Centro Médico Nacional Siglo XXI, Instituto Mexicano del Seguro Social, Ciudad de México, México

* Autor para correspondencia. Avenida Cuauhtémoc N. ${ }^{\circ} 330$, Colonia Doctores, Delegación Cuauhtémoc, CP 06720, Ciudad de México, México.

Correo electrónico: luisraulml@hotmail.com

(L.R. Meza-López).

http://dx.doi.org/10.1016/j.acmx.2017.01.006 1405-9940/

(c) 2017 Instituto Nacional de Cardiología Ignacio Chávez. Publicado por Masson Doyma México S.A. Este es un artículo Open Access bajo la licencia CC BY-NC-ND (http://creativecommons.org/ licenses/by-nc-nd/4.0/). 